\title{
THE RECURSIVE EQUIVALENCE TYPE OF A CLASS OF SETS ${ }^{1}$
}

\author{
BY J. C. E. DEKKER \\ Communicated by P. R. Halmos, February 28, 1964
}

1. Introduction. Let us consider non-negative integers (numbers), collections of numbers (sets) and collections of sets (classes). The letters $\epsilon$ and $o$ stand for the set of all numbers and the empty set of numbers respectively. We write $C$ for inclusion, proper or improper. A mapping from a subset of $\epsilon$ into $\epsilon$ is called a function; if $f$ is a function, we denote its domain and its range by $\delta f$ and $\rho f$ respectively. Let a class of mutually disjoint nonempty sets be called an $m d$-class; such a class is therefore countable, i.e., finite or denumerable. We recall that the recursive equivalence type (abbreviated: RET) of a set $\alpha$, denoted by $\operatorname{Req}(\alpha)$, is defined [1, p. 69] as the class of all sets which are recursively equivalent to $\alpha$. We wish to consider the problem: "How can we define the RET of an md-class in a natural manner?" Throughout this note $S$ stands for an md-class and $\sigma$ for the union of all sets in $S$; for every $x \in \sigma$ we denote the unique set $\alpha$ such that $x \in \alpha \in S$ by $\alpha_{x}$.

Definitions. A set $\gamma$ is a choice set of $S$, if

(1) $\gamma \subset \sigma$,

(2) $\gamma$ has exactly one element in common with each set in $S$.

The set $\gamma$ is a good choice set of $S$ (abbreviated: gc-set), if it also satisfies

(3) there exists a partial recursive function $p(x)$ such that $\sigma \subset \delta p$ and $(\forall x)\left[x \in \sigma \Rightarrow p(x) \in \gamma \cdot \alpha_{x}\right]$.

Consider the special case that the md-class $S$ is a finite class of finite sets. Then

(a) every choice set of $S$ is a good choice set,

(b) every two choice sets of $S$ are recursively equivalent,

(c) every two good choice sets of $S$ are recursively equivalent.

If the md-class $S$ is infinite, (a) and (b) need no longer be true. For let $S$ contain infinitely many sets of cardinality $\geqq 2$, e.g., $S=((0,1),(2,3),(4,5), \cdots)$. Then $S$ has $c$ choice sets. Every good choice set of $S$ has the form $p(\sigma)$ for some partial recursive function $p(x)$, hence $S$ has at most $\aleph_{0}$ good choice sets and (a) is false. Every nonzero RET contains exactly $\boldsymbol{\aleph}_{0}$ sets; the $c$ choice sets of $S$ can therefore not all be recursively equivalent and (b) is false. On the

1 This paper was written while the author was supported by a grant from the Rutgers Research Council. 
other hand, (c) still holds. For we have

Proposition P1. Every two good choice sets of an md-class are recursively equivalent.

Note that (a) does not even hold for every finite class consisting of two infinite sets. For let $S=\left(\tau, \tau^{\prime}\right)$, where $\tau$ and $\tau^{\prime}$ are complementary immune sets. Then $S$ has denumerably many choice sets, but if $S$ had a good choice set, $\tau$ and $\tau^{\prime}$ would be recursive. For every md-class $S$ we write $\zeta(S)$ for the class of all gc-sets of $S$. If $\zeta(S)$ is nonempty, $S$ is called a gc-class. The class $\left(\tau, \tau^{\prime}\right)$ mentioned above is an example of an md-class which is not a gc-class. P1 enables us to give the

Definition. For any gc-class $S$,

$$
\operatorname{RET}(S)=\operatorname{Req}(\gamma), \quad \text { for any } \gamma \in \zeta(S) \text {. }
$$

If $S$ is a finite md-class of finite sets, $S$ is a gc-class and $\operatorname{RET}(S)$ equals the cardinality of $S$. We need not exclude the trivial case that $S$ is empty, for then $\zeta(S)$ contains exactly one set, namely $o$.

2. Elementary properties. The sets $\alpha_{0}, \cdots, \alpha_{n}$ are separable if there exist mutually disjoint r.e. sets $\beta_{0}, \cdots, \beta_{n}$ such that $\alpha_{i} \subset \beta_{i}$ for $0 \leqq i \leqq n$. We write $\alpha_{0} \mid \alpha_{1}$ if $\alpha_{0}$ and $\alpha_{1}$ are separable.

Proposition P2. The finite md-class $S=\left(\alpha_{0}, \cdots, \alpha_{n}\right)$ is a gc-class if and only if $\alpha_{0}, \cdots, \alpha_{n}$ are separable; if $S$ is a gc-class, each choice set of $S$ is a gc-set and $R E T(S)$ equals the cardinality of $S$.

A gc-class is called isolated if each (or equivalently, at least one) of its gc-sets is isolated. In other words, a gc-class is isolated if its RET is an isol. For every nonempty gc-class $S$ we have: $\sigma$ is a finite set if and only if $S$ is a finite class of finite sets. Similarly,

Proposition P3. Let $S$ be a nonempty gc-class. Then $\sigma$ is an isolated set if and only if $S$ is an isolated class of isolated sets.

Two classes $S_{1}$ and $S_{2}$ with unions $\sigma_{1}$ and $\sigma_{2}$ respectively are separable if $\sigma_{1} \mid \sigma_{2}$. For any two classes $A$ and $B$ we write

$$
A \times B=\{j(\alpha \times \beta) \mid \alpha \in A \text { and } \beta \in B\},
$$

where $j(x, y)=x+(x+y)(x+y+1) / 2$.

Proposition P4. Let $S_{1}$ and $S_{2}$ be separable md-classes. Then $S_{1} \cup S_{2}$ is an md-class and

(a) $S_{1} \cup S_{2}$ is a gc-class if and only if both $S_{1}$ and $S_{2}$ are gc-classes,

(b) if $S_{1} \cup S_{2}$ is a gc-class, $R E T\left(S_{1} \cup S_{2}\right)=\operatorname{RET}\left(S_{1}\right)+\operatorname{RET}\left(S_{2}\right)$. 
Proposition P5. Let $S_{1}$ and $S_{2}$ be nonempty md-classes. Then $S_{1} \times S_{2}$ is a nonempty md-class and

(a) $S_{1} \times S_{2}$ is a gc-class if and only if both $S_{1}$ and $S_{2}$ are gc-classes,

(b) if $S_{1} \times S_{2}$ is a gc-class, $\operatorname{RET}\left(S_{1} \times S_{2}\right)=\operatorname{RET}\left(S_{1}\right) \cdot \operatorname{RET}\left(S_{2}\right)$.

3. The class $\operatorname{Bin}(\alpha)$. Let $\left\{\rho_{n}\right\}$ be the canonical enumeration of the class of all finite sets $[2$, p. 81$]$ and $r_{n}=$ cardinality of $\rho_{n}$. For any set $\alpha$ and any number $k$ we write

$$
C(\alpha, k)=\left\{n \mid \rho_{n} \subset \alpha \text { and } r_{n}=k\right\}, \quad \operatorname{Bin}(\alpha)=\{C(\alpha, k) \mid k \geqq 1\} .
$$

Note that $\operatorname{Bin}(\alpha)$ is an md-class for any set $\alpha$; if $\alpha$ is a finite set of cardinality $n$, the members of $\operatorname{Bin}(\alpha)$ are separable and $\operatorname{Bin}(\alpha)$ is a gc-class with $n$ as cardinality and RET. For any infinite set $\alpha$, $\operatorname{Bin}(\alpha)$ is a denumerable md-class of infinite sets; the next proposition tells us when $\operatorname{Bin}(\alpha)$ is a gc-class. We write $\operatorname{Req}(\epsilon)=R$ and refer to $[2$, pp. 80,84$]$ for the definition of a regressive set and a regressive isol.

Proposition P6. Let $\alpha$ be infinite and $A=\operatorname{Req}(\alpha)$. Then

(a) if $\alpha$ has an infinite r.e. subset, Bin $(\alpha)$ is a gc-class of RET $R$,

(b) if $\alpha$ is a regressive set, $\operatorname{Bin}(\alpha)$ is a gc-class of $R E T A$,

(c) if $\alpha$ is immune, but not regressive, Bin $(\alpha)$ is not a gc-class.

It follows that among the $c$ existing md-classes of immune sets, exactly $c$ are gc-classes and exactly $c$ are not. It is shown in [3] that though the collection $\Lambda_{R}$ of all regressive isols is not closed under addition one multiplication, one can extend the $\min (x, y)$ function from $\epsilon^{2}$ into $\epsilon$ in a natural manner to a $\min (X, Y)$ function from $\Lambda_{R}^{2}$ into $\Lambda_{R}$. However, $\min (X, Y)$ need no longer assume one of the values $X$ and $Y$.

Proposition P7. Let $\alpha, \beta$ be two nonempty isolated sets, $A=\operatorname{Req}(\alpha)$ $B=\operatorname{Req}(\beta)$ and

$$
S=\{j(\xi \times \eta) \mid(\exists n)[n \geqq 1 \text { and } \xi=C(\alpha, n) \text { and } \eta=C(\beta, n)]\} \text {. }
$$

If $\alpha$ and $\beta$ are regressive, i.e., $A, B \in \Lambda_{R}$ then $S$ is a gc-class with $R E T(S)$ $=\min (A, B)$.

It can be shown that $S$ may be a gc-class while the sets $\alpha$ and $\beta$ are immune, but not both regressive.

4. Characterization of gc-classes.

Definitions. Let $p(x)$ be a partial recursive function and $S$ a gc-class. Then $p(x)$ is a $g c$-function of $S$, if 
( $\alpha) \sigma \subset \delta p$ and $p(\sigma) \in \zeta(S)$,

$(\beta)(\forall x)\left[x \in \sigma \Rightarrow p(x) \in p(\sigma) \cdot \alpha_{x}\right]$,

$(\gamma) \rho p \subset \delta p$ and $(\forall x)\left[x \in \delta p \Rightarrow p^{2}(x)=p(x)\right]$

A gc-function is a partial recursive function which is a gc-function of at least one gc-class.

Every gc-class has at least one gc-function. For if a partial recursive function $p(x)$ is related to $S$ by $(\alpha)$ and $(\beta)$, then $p(x)$ has a restriction which satisfies $(\alpha),(\beta)$ and $(\gamma)$. With every partial recursive function $p(x)$ we associate the md-class $\operatorname{Gen}(p)=\left\{p^{-1}(y) \mid y \in \rho p\right\}$ of r.e. sets. This md-class is empty if and only if $p(x)$ is nowhere defined.

Proposition P8. A partial recursive function $p(x)$ is a gc-function if and only if it satisfies $(\gamma)$. Moreover, if $p(x)$ satisfies $(\gamma)$, it is a $g c$ function of the class $S=\operatorname{Gen}(p)$ with $\sigma=\delta p$ and $p(\sigma)=\rho p \in \zeta(S)$.

Proposition P9. Let $p(x)$ be a gc-function of the gc-class $S$. Then

$$
\delta p=\sigma \Leftrightarrow S=\operatorname{Gen}(p) .
$$

Definition I. A class $S$ is primitive, if it satisfies one of the three conditions: (i) $S$ is empty, (ii) $S$ is a nonempty, finite md-class of r.e. sets, (iii) $S$ is a denumerable md-class of r.e. sets and there exists a recursive function $a(n, x)$ such that if $\alpha_{n}=\rho a(n, x)$, then $S$ consists of the distinct sets $\alpha_{0}, \alpha_{1}, \cdots$.

Definition II. A class $S$ is primitive, if it is a gc-class with a gcfunction $p(x)$ such that $S=\operatorname{Gen}(p)$.

Definition III. A class $S$ is primitive, if $S=\operatorname{Gen}(p)$ for some partial recursive function $p(x)$.

Proposition P10. The three definitions of a primitive class are equivalent.

Corollary. A class $S$ is primitive if and only if it is a gc-class with a gc-function $p(x)$ such that $\delta p=\sigma$.

Definition. An md-class $T$ is a restriction of the gc-class $S$, if

(a) for every $\beta \in T$, there is an $\alpha_{\beta}$ such that $\beta \subset \alpha_{\beta} \in S$,

(b) there is a $\gamma \in \zeta(S)$ such that $\beta \in T \Rightarrow \gamma \cdot \alpha_{\beta} \subset \beta$.

Proposition P11. An md-class is a gc-class if and only if it is a restriction of some primitive gc-class.

While there are $c$ gc-classes, only $\boldsymbol{\aleph}_{0}$ of them are primitive. For each RET $A$ there exists a gc-class with $A$ as its RET, but a primitive class can only have one of $0,1, \cdots, R$ as its RET. The gc-sets of a primitive class $P$ are readily characterized. For if $P$ is finite, the gc-sets of $P$ are the choice sets of $P$, and if $P$ is infinite, say 


$$
P=\left(\alpha_{0}, \alpha_{1}, \cdots\right), \quad \alpha_{n}=\rho a(n, x),
$$

$a(n, x)$ a recursive function, then $\gamma \in \zeta(p)$ if and only if $\gamma=\rho a\left(f_{n}, u_{n}\right)$, for a recursive permutation $f_{n}$ and a recursive function $u_{n}$. Finally, the restrictions of any given primitive class can be simply described. Thus Proposition P11 serves a purpose.

\section{REFERENCES}

1. J. C. E. Dekker and J. Myhill, Recursive equivalence types, Univ. California Publ. Math. 3 (1960), 67-214.

2. J. C. E. Dekker, Infinite series of isols, Proc. Sympos. Recursive Function Theory, pp. 77-96, Amer. Math. Soc., Providence, R. I., 1962.

3. - The minimum of two regressive isols, Math. Z. 83 (1964), 345-366.

4. A. Nerode, Extensions to isols, Ann. of Math. (2) 73 (1961), 362-403.

RUtgers, the State University 